\title{
Novel in situ gel-forming solid dosage form (gfSDF) prepared by simple syringe- based moulding: a screening study
}

Margherita Falavigna ${ }^{\mathrm{a}, \mathrm{b}}$, Nataša Škalko-Basnet ${ }^{\mathrm{a}}$ Cristina Cavallari $^{\mathrm{b}}$, Andrea Pini ${ }^{\mathrm{b}}$, Barbara Luppi ${ }^{\mathrm{b}}$, Massimiliano Pio di Cagno ${ }^{\mathrm{a}, *}$

${ }^{a}$ Drug Transport and Delivery Research Group, Department of Pharmacy, University of Troms $\phi$ The Arctic University of Norway, Troms $\phi$, Norway

${ }^{\mathrm{b}}$ Drug Delivery Research Group, Department of Pharmacy and Biotechnology, University of Bologna, Bologna, Italy

*Corresponding author: Massimiliano Pio di Cagno; Tel: +47 776 45301; e-mail: Massimiliano.p.cagno@uit.no

\begin{abstract}
The aim of this study was to prepare and optimize a novel type of in situ gel-forming solid dosage form (gfSDF) to be used in the treatment of mucosal/skin ulcerations. For this purpose, a simple but reliable syringe-based hot melt/moulding method (a very simplistic method reassembling hot melt extrusion (HME) in small scale) was employed. Chloramphenicol (antibiotic) and ibuprofen (antiinflammatory) were chosen as model active pharmaceutical ingredients (APIs) to be loaded into the gfSDFs. To optimize the formulations, the gfSDFs of different compositions were studied in terms of APIs release from the matrix, solid-state characteristics, gellification properties and gfSDFs resistance to mechanical stress. Release studies showed that both APIs were released at a constant rate at different $\mathrm{pH}$ (pH 5 and 7.4, respectively) and the changes in the formulation composition affected the release behaviour. Differential scanning calorimetry (DSC) results evidenced the complete solubilization of both API in the solid matrix. Texture analysis showed that the gfSDFs were capable of swelling once in a contact with aqueous environment and that the textural properties changed extemporaneously from the solid to gel form. The gel formed after hydration exhibited high cohesiveness and adhesiveness, an indication of good mucoadhesion properties. Friability testing confirmed satisfactory physical strength for a solid dosage form.
\end{abstract}

\section{Keywords}

Chloramphenicol; ibuprofen; solid dosage form; differential scanning calorimetry (DSC); in situ gelling; drug release; mucoadhesion 


\section{Introduction}

Ulcer is the common term used to indicate chronic sores and lesions of multifactorial origin that can affect skin and mucous membranes, accompanied by tissue damaging and disintegration (Hermans, 2010). Ulcerations can occur in skin, as well as in different mucosal tissues of the body such as gastrointestinal tract (Vasopolli et al., 2016), rectum (Chang et al., 2006) and genital mucosa (Lehman et al., 2010). Ulcerations and mucosal wounds can be caused by autoimmune disorders (e.g. Crohn's disease, (Bandzar et al., 2013)), specific syndromes (e.g. diabetes), environmental causes (e.g. skin laceration) or directly caused by microbiological infection and tissue colonization (i.e. Helicobacter pylori in the gastric ulcer (Nomura et al., 1994) or Treponema pallidum in primary syphilis (Singh and Romanowski, 1999). Standard treatments for ulceration can vary accordingly to accessibility to the sores and cause of the ulceration and they might comprise wound protection by dressing (Boateng and Catanzano, 2015) as well as antibiotic/antiseptic local/systemic treatment (Howell-Jones et al., 2005). Symptomatic effects such as itching and pain sensation are often associated with sores and ulcerations and can be controlled by local administration of anti-inflammatory medications (Jøraholmen et al., 2015; Milani and Iacobelli, 2012). Skin and mucosal ulcerations remain very difficult to treat with current therapy, especially when the damage to the tissue occurs in region of the body of difficult accessibility (i.e. rectal, gastrointestinal and vaginal mucosal ulcerations) and/or when the sores are associated to microbiological infections (Fryberg and Banks, 2015).

The tentative approach of this study was to design a solid dosage formulation capable of extemporaneously dressing the sores and co-administer an antibiotic together with an antiinflammatory drug. Ideally, the formulation should provide wound protection together with antibiotic and anti-inflammatory effect for a prolonged period.

For this purpose, a simple syringe-based hot melt moulding method (considered a very simplified version of the well-established HME (Patil et al., 2016)) has been developed and employed in order to obtain gel-forming solid dosage form (gfSDF) capable of extemporaneous gellification once hydrated. The manufacturing process employed was based on the preparation of a fluid paste of hydrophilic polymers containing one API, a plasticizer in which a second drug was solubilized and subsequent moulding.

PEG 4000 was chosen as a principal constituent of the gfSDF due to its relatively low melting temperature (Vasa et al., 2014) and good miscibility properties with other high tolerable gel-forming hydrophilic polymer such as hydroxypropyl methylcellulose (HPMC) and hydroxypropyl cellulose (HPC) (Janssens et al., 2008; Joshi, 2011; Wang and Tang, 2008). Tween 20 (polysorbate 20) was 
chosen as the plasticizer in order to increase the fluidity of the hot paste during syringe extrusion and to act as solubilizer for poorly soluble drugs (HLP of 16.7).

Chloramphenicol (CHL) was chosen as a model of antibiotic drug (Fig. 1 reports the chemical structure of CHL together with some of its physicochemical properties (Drugbank, 2017a; Lombardo et al., 2001) based on its broad antimicrobial spectrum against Gram-positive and -negative bacteria. This API is commercially available as a topical cream for eye and ear infections; however, it could be a potential antibiotic for mucosal infections as well (Heal et al., 2009).

Ibuprofen (IBU) was chosen as model of anti-inflammatory drug (Fig. 1 reports its chemical structure together with some of its physicochemical properties (Drugbank, 2017b; Stein et al., 2011)) because of its well-known, effective, safe and well-tolerated anti-inflammatory activities (Pierce and Voss, 2010). In this work, shape of the solid dosage form as well as the drugs chosen makes this formulation most suited for vaginal delivery, but the method can be easily adapted in order to obtain tablet (for oral use), suppositories (for rectal therapy) as well as thin sheets (for plaster in skin therapy) varying also the choice of the APIs.

\section{Materials and methods}

\subsection{Materials}

CHL (Fig. 1) was purchased from Norks Medisinaldepot (Oslo, Norway).

IBU (Fig. 1) was obtained from Fluka (Sigma-Aldrich GmbH Chemie, Steinheim, Germany). Hypromellose 4000 (hydroxypropyl methylcellulose, HPMC) was obtained from Apotekproduksjon AS (Oslo, Norway). Polyethylene glycol 4000 (PEG) and hydroxypropyl cellulose (HPC, mw 80000) were obtained from Sigma-Aldrich Chemie GmbH (Steinheim, Germany) and Tween 20 (polysorbate 20) was purchased from ICI Surfactants (Everberg, Belgium). Di-sodium hydrogen phosphate dihydrate $\left(\mathrm{Na}_{2} \mathrm{HPO}_{4} \cdot 2 \mathrm{H}_{2} \mathrm{O}\right)$, sodium di-hydrogen phosphate monohydrate $\left(\mathrm{NaH}_{2} \mathrm{PO}_{4} \cdot \mathrm{H}_{2} \mathrm{O}\right)$ (both chemicals purchased from Merck KGaA, Darmstadt, Germany) and sodium hydroxide ( $\mathrm{NaOH}$, Sigma-Aldrich) were used for the preparation of the neutral ( $\mathrm{pH} 7.4, \mathrm{pH}$ meter Lab 744 Metrohm AG, Herisau, Switzerland) phosphate buffer saline (PBS). Acetic acid ( $\geq 99.8 \%$ ) and sodium acetate trihydrate (Sigma-Aldrich) were used in order to prepare the acetate buffer saline (pH 5). Methanol (Sigma-Aldrich) was employed for the preparation of the mobile phase. All chemical were of analytical grade.

\subsection{Methods}

2.2.1 Preparation of the gel-forming solid dosage forms (gfSDFs) 
For the preparation of the gfSDFs, a syringe-based hot-melt moulding method was developed and employed. Briefly (Fig. 2), the method consisted of the preparation of a moderate-heated paste (65 ${ }^{\circ} \mathrm{C}$, primary mixture) composed of polyethylene glycol, small amount of cellulose derivatives (HPMC or HPC) and one API. Temperature of $65^{\circ} \mathrm{C}$ was selected in order to be slightly above the melting point of PEG $4000\left(63.5^{\circ} \mathrm{C}\right.$, Figure 3). Simultaneously, a solution of a second API in a plasticizer (surfactant solution) was prepared. The surfactant solution was added to the warm paste under continuous stirring, to obtain a homogeneous viscous mixture. The last step of the preparation was the moulding of the warm fluid mixture into shaped dies. Two different types of the gfSDFs were prepared (the compositions reported in Table 1). The first formulation type ( $\mathrm{S} 1$ or $\mathrm{S} 1^{*}$ ) was obtained by gently adding a solution of CHL in Tween 20 (surfactant solution) to primary mixture containing IBU under stirring condition. The second formulation type (S2 or S2*) was prepared by the same procedure but in this case, IBU was dissolved in Tween 20 (plasticizer), whereas CHL was placed in the primary mixture. For both formulations, the final mixture was kept in a water bath at moderate temperature $\left(65^{\circ} \mathrm{C}\right)$ for 15 min under stirring to achieve a homogeneous fluid mixture. Finally, the warm fluid paste was transferred into a pre-heated plastic syringe (approx. $65^{\circ} \mathrm{C}$ ), poured into tabletshaped dies (1.2 cm diameter), and let to cool for $60 \mathrm{~min}$.

\subsubsection{Differential scanning calorimetry (DSC)}

In order to characterize the changes to the solid state of the APIs as well as the excipients in the gfSDF after formulation, DSC analysis were performed. Thermograms were obtained employing a Mettler-Toledo (Mettler-Toledo GmbH, Geissen, Germany) instrument equipped with a FP 80HT control unit and a FP 85TA cell furnace. Samples (approx. $10 \mathrm{mg}$ ) were analyzed in the range 30$200^{\circ} \mathrm{C}$, at an increasing heating rate of $10^{\circ} \mathrm{C} / \mathrm{min}$.

\subsubsection{Release study of chloramphenicol and ibuprofen from the gfSDF}

The gfSDF was inserted in a dialysis membrane (molecular weight cut-off 12-14 kDa, Medicell Membranes Ltd, London, UK) and placed in a beaker containing $50 \mathrm{~mL}$ of buffer. All the experiments were carried out under stirring at room temperature $\left(23-25^{\circ} \mathrm{C}\right)$. The release of both drugs from the gfSDF was studied at pH 5 (acetate buffer: acetic acid, sodium acetate trihydrate) and at pH 7.4 (PBS: di-sodium hydrogen phosphate dihydrate, sodium di-hydrogen phosphate monohydrate, sodium hydroxide). Samples (1 mL each) were withdrawn at regulate time intervals $(0.5,1,2,3,5,8$ and 24 h) and replaced with equal volumes of buffer to maintain the sink conditions. To determine the total drug loaded in the gfSDF, the formulation residue (after 24 hours) was removed from the dialysis membrane and placed in an ultrasound bath for $60 \mathrm{~min}$ (Branson ultrasonic cleaner 5510E-MT, 
Danbury, USA). The obtained dispersion was vigorously stirred for $30 \mathrm{~min}$ and finally bath sonicated for additional $60 \mathrm{~min}$ to ensure total dissolution of the APIs in the media. Both formulations were tested in triplicates.

To enable the simultaneous quantification of both drugs, a dedicated HPLC method was developed. Samples obtained from the release studies were analyzed by high precision liquid chromatography (HPLC) using a Waters 2690 Separation Module HPLC system, equipped with Waters 996 Photodiode Array Detector (Waters Corporation, Milford, USA). Chromatographic separation of the two drugs was conducted using a Waters XTerra RP18 $5 \mu \mathrm{m} 3.9 \times 150 \mathrm{~mm}$ reverse phase column, using a mobile phase composed of methanol (60\%) and water (40\%) with $0.1 \%$ of acetic acid (flow rate of $0.8 \mathrm{~mL} / \mathrm{min}$, injection volume $10 \mu \mathrm{L}$ ). CHL was detected at the wavelength of $278 \mathrm{~nm}$ (Satinski et al., 2006) and IBU at $264 \mathrm{~nm}$ (Stein et al., 2011; Zhu et al., 2005). Calibration curves for both drugs were prepared at their specific wavelengths of detection (coefficient of determination $R^{2} \geq 0.999$ ).

\subsubsection{Texture analysis}

Three replicates of each gfSDF ( $\mathrm{S} 1$ and $\mathrm{S} 2$ ) were tested at $\mathrm{pH} 5$ and 7.4, respectively, using a TA.XT.Plus Texture Analyzer (Stable Micro Systems Ltd., Surrey, UK). The method was adapted from Yang et al. (1998). Briefly, $1 \mathrm{~mL}$ of PBS was placed on top of the gfSDF and changes in texture as well as gel layer thickness were measured over time $(0.5,1,2,3$ and $5 \mathrm{~h})$. The experiments were carried out with a steel mucoadhesion probe with a diameter of $10 \mathrm{~mm}$ (starting position $4 \mathrm{~mm}$ from sample holder; pre-test speed $2 \mathrm{~mm} / \mathrm{s}$, test speed $0.2 \mathrm{~mm} / \mathrm{sec}$, force 500 gram-force, trigger force 0.7 gram-force). Data were analyzed by Texture Exponent software (Texture Technologies Corporation and Stable Micro Systems Ltd., Hamilton, MA, USA) at a rate of 200 points per seconds.

Parameters such as the cohesiveness, hardness and adhesiveness were determined from the resultant force-time plot (Fig. 4). Hardness (expressed in gram-force) is the force required to compress the sample, cohesiveness (expressed in gram-force*seconds) is the momentum of the deformation before breakage (positive area) and adhesiveness is the momentum necessary to pull the probe away from the sample (negative area, gram-force*s). These parameters enable the estimation of eventual bioadhesion.

\subsubsection{Friability test}

Three replicates for each formulation (S1 and S2) were weighted on an analytical balance. Samples were subsequently placed in the drum of an Erweka Tar 20 friability tester (Erweka GmbH, Heusenstamm, Germany) rotating at $25 \mathrm{rpm}$ for 4 minutes (total 100 rotations; European (Pharmacopoeia, 2010)). At the end of the rotation time, each sample was cleaned from eventual dust 
and re-weighed to calculate the mass loss after the experiment. The percentage of mass loss was calculated using equation 1 :

$$
\text { Mass loss }(\%)=\frac{\text { Final mass } g f S D F}{\text { Initial mass } g f S D F} \times 100
$$

A mass loss below $1 \%$ was considered acceptable.

\subsubsection{Statistical analysis}

Student's t-test was performed to evaluate significant differences between formulations and sample sets. $\mathrm{P} \leq 0.05$ was considered significantly different.

\section{Results}

\subsection{Preparation method of the gfSDFs}

The method developed end employed in this work for the preparation of the gfSDFs is summarized in Fig. 2. The method provided a fast, convenient and reliable technique that permitted the incorporation of both CHL and IBU in the gfSDFs. Different types of gfSDFs were prepared (Table 1) employing different procedures for drug loading into the melted paste. In fact, the two APIs could be added in the primary mixture as well as in the surfactant solution. The method was suitable for all types of gfSDFs and the developed technique permitted the preparation of multiple units per a batch. The relatively low temperature used during the process $\left(65^{\circ} \mathrm{C}\right)$ allowed formation of a homogeneous fluid paste that was easy to pour from the pre-heated syringe into the tablet-shaped dies with good precision. The produced gfSDFs were of identical shape and similar weight.

\subsection{Differential scanning calorimetry}

In Fig. 3, the thermogram of one gfSDF $\left(\mathrm{S}_{2} *\right)$ is reported as exemplar together with the thermogram of the principal components contained in the formulation (PEG 4000, CHL and IBU). PEG 4000 appeared to be crystalline at the solid state, with a clear melting point and corresponding endothermic peak at $63.5{ }^{\circ} \mathrm{C}$. IBU and $\mathrm{CHL}$ also showed sharp endothermic peaks at $78.1{ }^{\circ} \mathrm{C}$ and $151.9{ }^{\circ} \mathrm{C}$ respectively, indication of high degree of crystallinity. Differently, the thermogram of the gfSDF did not evidence any endothermic peak in correspondence of these two temperatures. Moreover, the endothermic peak corresponding to PEG 4000 resulted less sharp and shifted to lower temperature (maximum at approx. $60^{\circ} \mathrm{C}$ ) indication of the occurrence of partial amorphization. 


\subsection{Release of ibuprofen and chloramphenicol from the gfSDFs}

The release profiles of IBU and CHL from different formulations ( $\mathrm{S} 1$ and $\mathrm{S} 2$ ) at different $\mathrm{pH}$ environments (5.0 and 7.4) are reported in Fig. 5. Release of both drugs at pH 5.0 is reported in graph A, whereas, the release at $\mathrm{pH} 7.4$ is reported in graph B. Both APIs could be detected in the bulk solution already after $30 \mathrm{~min}$ and the released amount gradually increased over time.

The quantification method developed permitted the detection of CHL and IBU in the same chromatographic run, with retention times of $3 \mathrm{~min}$ (at $278 \mathrm{~nm}$ ) for CHL and $14 \mathrm{~min}$ (at $264 \mathrm{~nm}$ ) for IBU, with a good resolution (Rs > 4).

As it can be seen in Fig. 5A, the release of IBU at pH 5.0 was incomplete after $24 \mathrm{~h}$ (approx. 40\%, Figure 5A), whereas CHL was substantially completely released after this time interval (80-100\%). For both drugs, a linear increase of percentage drug release with time was obtained in the first $5 \mathrm{~h}$, leading to a constant drug release rate. It is noteworthy that at acidic $\mathrm{pH}, \mathrm{CHL}$ is released faster than IBU (i.e. higher slope of the linear regression curve). Moreover, both drugs did not exhibit a significant difference $(\mathrm{P}>0.05)$ in the release profiles of both types of gfSDFs (S1 or S2) after $24 \mathrm{~h}$. At $\mathrm{pH} 7.4$, it appears that there is a significant difference $(\mathrm{P}<0.05)$ between formulation $\mathrm{S} 1$ and $\mathrm{S} 2$ after 24 hours. In fact, both drugs were completely released from the formulation S2, whereas, only $70 \%$ of the both APIs was released from the formulation S1. No significant differences in release kinetics were observed for the gfSDF prepared with HPC ( $\mathrm{S}^{*}$ and $\mathrm{S} 2 *$, data not shown) in comparison to the ones containing HPMC (S1 and S2).

\subsection{Texture properties of gfSDFs}

The core-gel interface resulting from the interaction between the gfSDFs and the buffer has been detected by measuring the changes in textural and physical/mechanical properties associated with changes in the gel layer growth.

Fig. 6 represents the force-displacement profiles of gfSDFs with different extents of hydration. The mucoadhesion probe that penetrated into the formulation faced a resistance force, which is a function of the travelled distance into the gel and gel texture/strength. This profile is comparable with the one obtained by Yang et al. (1998) and indicates how the dry sample (A) leads to a higher slope and corresponds to a hard consistency, whereas a lower slope was due to a weaker gel strength, which corresponded to the gfSDFs samples that had been hydrated over time (B, C, D, E, F). Fig. 7 gives a visual evidence of the changes in the consistency of the formulation under hydration over time (extemporaneous gel formation from gfSDF). The cohesiveness and gel layer thickness results (Fig. 8) confirmed that both formulations ( $\mathrm{S} 1$ and S2) were able to increase their cohesiveness once in a contact with buffer over time (pH 5.0 and 7.4) and that all of the tested samples followed the same 
pattern regarding the changes in the texture. A gradual increase in a gel layer thickness over time is shown for both formulations at $\mathrm{pH} 5.0$ and $\mathrm{pH} 7.4$, respectively. These changes occurred primarily in the first 3 hours, whereas a plateau was reached after $5 \mathrm{~h}$, suggesting the maximum grade of swelling and hydration of the formulations at those conditions.

All gfSDFs were found to express adhesive properties (Fig. 4, negative area) soon after the contact with the buffer.

\subsection{Friability of gfSDFs}

Friability was measured to define the physical strength of gfSDFs after exposure to mechanical stress. The maximum mass loss considered acceptable was 1\% (European Pharmacopoeia, 2010). For formulation $\mathrm{S} 1$ a loss of $0.045 \% \pm 0.001$ was determined, whereas for formulation $\mathrm{S} 2$, the average weight loss was slightly higher $(0.129 \% \pm 0.002)$ but still in the range of acceptance.

\section{Discussion}

The rationale behind the preparation of this new formulation was to produce a gel-forming solid dosage form (gfSDF) able to co-administer and sustain the release of an antibiotic and an antiinflammatory drug, once in contact with moisturised environments (i.e. mucosal tissues).

For the preparation of the gfSDF, PEG 4000 was chosen as major component of the mixture due to its relatively low melting point (Fig. 3) and good miscibility with cellulose derivatives such as HPMC and HPC (Janssen et al., 2008). In this work, a PEG/cellulose ratio of 2:1 was found optimal in order to obtain a fluid homogeneous mixture at $65^{\circ} \mathrm{C}$.

The preparation method for the gfSDFs resulted a time-effective, reliable and reproducible technique that permitted a proper incorporation of both drugs. Moreover, DSC results (Fig. 3) clearly evidenced the complete solubilization of the both APIs in the solid polymeric matrix.

Different types of gfSDFs (Table 1) were prepared and studied in order to evaluate whether the release of each drug would be affected by the mixing order, such as its direct incorporation in the primary mixture or by its previous dissolution into a plasticizer.

To evaluate drug release from the gfSDFs, the formulations were placed in dialysis tube with a cutoff size of 11-12 kDa. The cut-off size of the membrane was chosen in order to contain eventual micelles and aggregate (e.g. Tween micelles) that could entrap some drug inside, hampering a proper quantification of the drug release. In fact, in this way, only the fraction of a freely dissolved API should be measured whereas the eventual fraction of APIs contained in a form of micelles or aggregates (i.e. Tween 20) should remain confined within the dialysis tube. 
The novel quantification method for IBU and CHL permitted an accurate and timesaving detection of both drugs simultaneously with good resolution (Rs $>4$ ).

Release studies (Fig. 5) showed that the formulation allows a sustained release of both APIs. It has been reported before that PEG 4000 was suited for the preparation of solid dosage form by extrusion, for immediate drug release (Perissutti et al., 2002). In this case, the addition of cellulose derivatives allows better control of drug release. In fact, the gfSDFs were able to release APIs at a constant release rate for the first $5 \mathrm{~h}$ of the experiment (linear regression of the release curves). The experiment at $\mathrm{pH} 7.4$ (Fig. 5B) showed that the release after $24 \mathrm{~h}$ was almost complete for both drugs from formulation S2, whereas S1 was retaining both APIs to a higher extent (approx. 30\% of unreleased APIs after 24 hours). This difference is an indication that the composition of the gfSDF affects the drug release. At pH 5.0, it appeared that the physicochemical properties of the two different APIs, rather than formulation characteristics, affected the release profiles. This is of a great importance when applying the novel system for incorporation of different drug combinations. In acidic condition (pH 5.0, Fig. 5A) only CHL was completely released after $24 \mathrm{~h}$, whereas approx. only $40 \%$ of IBU was released from the gfSDF. This result is not surprising and is related to the known dependence of IBU solubility on the environment pH ( $\mathrm{Li}$ et al., 2008; Shaw et al., 2005). IBU has a much lower water solubility at acidic $\mathrm{pH}$ than at neutral/basic $\mathrm{pH}$ ( $\mathrm{pKa}$ of 4.9), thus hampering its complete release in the bulk solution. It should be also noted that there was no significant difference between the release of IBU and CHL from formulation S1 and S2 at pH 5.0. The employment of a different type of cellulose derivative (HPC, formulation $\mathrm{S}^{*}$ and $\mathrm{S} 2 *$ ) did not significantly affected drug release kinetics (data not shown).

Gel layer thickness together with cohesiveness are the key parameters that affect drug release from the swelling-controlled matrix systems (Harland et al., 1988). Results from texture analysis (Fig. 6 and Fig. 7) showed that the formulations were able to form a gel layer once in a contact with the buffer and change their gel layer thickness and cohesiveness over time. In fact, the distance between the periphery of the outer surface and the core/gel interface increased with the grade of hydration of the gfSDFs. Even though it seemed that the two formulations (S1 and S2) had comparable cohesiveness at $\mathrm{pH} 5.0$ (Fig. 8A), it appeared that at $\mathrm{pH} 7.4$ the cohesiveness profile of the two formulations were slightly different (Fig. 8B, black symbols). Specifically, formulation S1 showed a tendency of higher cohesiveness after 2-3 hours in comparison to S2. Even though it is difficult to derive definitive conclusions due to the high standard deviations identified, these results are in agreement with the higher release rate of both drugs at $\mathrm{pH} 7.4$ from formulation type $\mathrm{S} 2$ in comparison to S1 (Figure 5). 
All gfSDFs tested exhibited adhesive properties (Fig. 4, negative area) soon after the contact with the buffer, but changes in adhesiveness were not properly quantifiable because some samples had the tendency to stick to the mucoadhesion probe (clear indication of strong adhesiveness), hampering exact measurement. This is in agreement with previous findings that hydrophilic polymers such as PEG and cellulose derivatives become adhesive after hydration (Bottenberg et al., 1991). Another parameter that is essential for a development of solid dosage forms is the system's hardness/friability. Friability test indicated that the gfSDFs were able to resist mechanical stress without evidence of damage, lamination, capping or breakage, thus leading to the assumption that they could be suited for industrial packaging.

\section{Conclusions}

In this work, a novel type of gel-forming solid dosage form has been successfully developed. The preparation method for the gfSDFs provided a fast and reliable method to prepare the gfSDFs for skin/mucosal moisture-gelling and consequent local drug release, suitable for a screening study. The novel system could successfully permit incorporation of a combination of drugs, in this case IBU and CHL. Solid state investigation confirmed that both APIs were completely dissolved in the solid matrix, allowing extended release of both IBU and CHL over time (8 hours) at different $\mathrm{pH}$ (5.0 and 7.4, respectively). The gfSDFs exhibited satisfactory textural properties in terms of the gellification, cohesiveness and adhesiveness.

Moreover, these gfSDFs showed satisfactory resistance to mechanical stress making them suitable for further manufacturing handling process (e.g. packaging).

In conclusion, gfSDF appears to be a novel promising drug delivery system suitable for multidrug administration for the treatment of skin/mucosal ulcerations.

\section{Acknowledgement}

The authors would like to thank the European Union student exchange program Erasmus Plus for providing the mobility grant to researcher Margherita Falavigna and master student Matteo Capuano for his support during the manufacturing stage of the gfSDF.

\section{Conflict of interest}

The authors report no conflict of interest. 


\section{References}

Bandzar, S., Gupta, S., Platt, M.O., 2013. Crohn's disease: a review of treatment options and current research. Cell. Immunol. 286, 45-52.

Boateng, J., Catanzano, O., 2015. Advanced therapeutic dressings for effective wound healing-A review. J. Pharm. Sci. 104, 3653-3680.

Bottenberg, P., Cleymaet, R., de Muynck, C., Remon, J.P., Coomans, D., Michotte, Y., Slop, D., 1991. Development and testing of bioadhesive, fluoride-containing slow-release tablets for oral use. J. Pharm. Pharmacol. 43, 457-464.

Chang, J., Changchien, C., Chen, J., 2006. Solitary rectal ulcer syndrome An endoscopic and histological presentation and literature review. Int. J. Colorectal. Dis. 21, 348-356.

Drugbank database, http://www.drugbank.ca/drugs/DB00446 (accessed 01.03.2017).

Drugbank database, http://www.drugbank.ca/drugs/DB01050 (accessed 01.03.2017).

European Pharmacopoeia: Supplement 6.6 (published June 2009, official January 2010), Friability of $\begin{array}{lll}\text { uncoated } \quad \text { 01/2010:20907). } & \text { (reference }\end{array}$ http://www.ema.europa.eu/docs/en_GB/document_library/Scientific_guideline/2010/01/WC500044 297.pdf (last accessed October 2016).

Fryberg, R.G, Banks, J., 2015. Challenges in the Treatment of Chronic Wounds. Ad. Wound Care 4, 560-582.

Harland, R.S., Gazzaniga, A., Sangalli, M.E., Colombo, P., Peppas, N.A., 1988. Drug/polymer matrix swelling and dissolution. Pharm. Res. 5, 488-494.

Heal, C.F., Buttner, P.G., Cruickshak, R., Graham, D., Browning, S., Pendergast, J., Drobetz, H., Gluer, R., Lisec, C., 2009. Does single application of topical chloramphenicol to high risk sutured wounds reduce incidence of wound infection after minor surgery? Prospective randomised placebo controlled double blind trial. BMJ 338, a2812. 
Hermans, M.H., 2010. Wounds and ulcers: back to the old nomenclature. Wounds 22, 289-293.

Howell-Jones, R.S., Wilson, M.J., Hill, K.E., Howard, A.J., Price, P.E., Thomas, D.W., 2005. A review of the microbiology, antibiotic usage and resistance in chronic skin wounds. J. Antimicrob. Chemother. 55, 143-149.

Janssens, S., Denivelle, S., Rombaut, P., Van den Mooter, G., 2008. Influence of polyethylene glycol chain length on compatibility and release characteristics ternary solid dispersions of itraconazole in polyethylene glycol/ hydroxypropyl methylcellulose 2910 E5 blends, Eur. J. Pharm. Sci. 35, 203210.

Joshi, S.C., 2011. Sol-Gel Behaviour of hydroxypropyl methylcellulose (HPMC) in ionic media including drug release. Materials 4, 1861-1905.

Jøraholmen, M.W., Škalko-Basnet, N., Acharya, G., Basnet, P., 2015. Resveratrol-loaded liposomes for topical treatment of the vaginal inflammation and infections. Eur. J. Pharm. Sci. 79, 112-121.

Lehman, J.S., Bruce, A.J., Wetter, D.A., Ferguson, S.B., Rogers III, R.S., 2010. Reactive nonsexually related acute genital ulcers: Review of cases evaluated at Mayo Clinic. J. Am. Acad. Dermatol. 63, 44-51

Li, H., Hardy, R.J., Gu, X., 2008. Effect of drug solubility on polymer hydration and drug dissolution from polyethylene oxide (PEO) matrix tablets. AAPS PharmSciTech. 9437-443.

Lombardo, F. , Shalaeva, M.Y. , Tupper, K.A., Gao, F., 2001. ElogD oct: a tool for lipophilicity determination in drug discovery. 2. Basic and neutral compounds. J. Med. Chem. 44, 2490-2497.

Milani, M., Iacobelli, P., 2012. Vaginal use of ibuprofen isobutanolammonium (Ginenorm): efficacy, tolerability, and pharmacokinetic data: a review of available data. ISRN Obstet. Gynecol. 2012, 1-6.

Nomura, A., Stemmermann, G.N., Chyou, P., Perez-Perez, G.I., Blaser, M.J., 1994. Helicobacter pylori infection and the risk for duodenal and gastric ulceration. Ann. Intern. Med. 120, 977-981. 
Perissutti, B, Newton, J.M., Podczeck, F., Rubessa, F., 2002. Preparation of extruded carbamazepine and PEG 4000 as a potential rapid release dosage form. Eur. J. Pharm. Biopharm. 53, 125-132

Patil, H., Tiwari, R.V., Repka, M.A., 2016. Hot-Melt Extrusion: from theory to application in pharmaceutical formulation. AAPS PharmSciTech. 17, 20-42.

Pierce, C.A., Voss, B., 2010. Efficacy and safety of ibuprofen and acetaminophen in children and adults: a meta-analysis and quantitative review. Ann. Pharmacother. 44, 589-506.

Satínsky, D., Chocholous, P., Salabová, M., Solich, P., 2006. Simple determination of betamethasone and chloramphenicol in a pharmaceutical preparation using a short monolithic column coupled to a sequential injection system. J. Sep. Sci. 29, 2494-2499.

Shaw, L.R., Irwin, W.J., Grattan, T.J., Conway, B.R., 2005. The effect of selected water-soluble excipients on the dissolution of paracetamol and ibuprofen. Drug Dev. Ind. Pharm. 31, 515-525.

Singh, A.E., Romanowski, B., 1999. Syphilis: Review with emphasis on clinical, epidemiologic, and some biologic features. Clin. Microbiol. Rev. 12, 187-209.

Stein, P.C., di Cagno, M., Bauer-Brandl, A., 2011. A novel method for the investigation of liquid/liquid distribution coefficient and interface permeabilities applied to the water-octanol-drug system, Pharm. Res., 2140-2146.

Vasa, D.M., Dalal, N., Katz, J.M., Roorwani, R., Nevrekar, A., Patel, H., Buckner, I.S., Wildfong, P.L.D., 2014. Physical characterization of drug: polymer dispersion behavior in polyethylene glycol 4000 solid dispersions using a suite of complementary analytical techniques. J. Pharm. Sci. 103, 2911-2923.

Vasopolli, R., Malfertheiner, P., Kandulski, A., 2016. Helicobacter pylori and non-malignant upper gastrointestinal diseases. Helicobacter 21, 30-33.

Wang, L., Tang, X.A., 2008. A novel ketoconazole bioadhesive effervescent tablet for vaginal delivery: design, in vitro and in vivo evaluation. Int. J. Pharm. 350, 181-187. 
Yang, L., Johnson, B., Fassihi, R., 1998. Determination of continuous changes in the gel layer thickness of poly(ethylene oxide) and HPMC tablets undergoing hydration: a texture analysis study, Pharm. Res. 15, 1902-1906.

Zhu, Y., Shi, J., Li, Y., Chen, H., Shen, W., Dong, X., 2005. Storage and release of ibuprofen drug molecules in hollow mesoporous silica spheres with modified pore surface. Micropor. Mesopor. Mat. $85,75-81$. 